\title{
Exóstosis postraumática del quinto metacarpiano: Diagnóstico diferencial con osteocondroma
}

\author{
S. AMILlo ${ }^{(1)}$, L. M. RoMero ${ }^{(2)}$ \\ CLIINICA UNIVERSIDAD DE NAVARRA \\ (I) Doctor en Medicina. Especialista en CiRUGía de la Mano. Departamento de Cirugía ORtopédica Y \\ TRAUMATOLOGÍA \\ (2) Médico Residente. Departamento De Cirugía Ortopédica Y Traumatología
}

\section{Correspondencia}

Dr. Santiago Amillo Garayoa

Departamento de Cirugía Ortopédica y Traumatología. Clínica Universidad de Navarra

Avenida de Pío XII, 36

31008. Pamplona

Teléfono: 948255400

Fax: 948296500

e-mail: samillo@unav.es

\begin{abstract}
Un traumatismo directo en dorso de falanges o metacarpianos puede lacerar el periostio y generar un hematoma subperióstico que posteriormente se osifica, mostrando en estudios radiográficos una lesión extracortical osificada denominada en literatura inglesa como «turret exostosis» sin traducción. Debido al escaso número de «turret exostosis» de región metacarpiana en la literatura y al diagnóstico diferencial con osteocondroma presentamos un nuevo caso.
\end{abstract}

Palabras clave: Exóstosis, metacarpiano, traumatismo, osteocondroma.
Direct injury to the dorsum of the phalanges or metacarpal is able to produce a laceration of the periostium that forms a subperiosteal hematoma, which subsequently undergoes ossification, $\mathrm{X}$ ray show an ossified extracortical lesion called «turret exostosis». On account of the few cases reported in the orthopaedic literature and the need to make differential diagnosis with osteochondroma, we report a new case.

Key words: Metacarpal exostosis, trauma, osteochondroma.

\section{INTRODUCCIÓN}

U $\mathrm{n}$ traumatismo directo en falanges o metacarpianos puede llevar a la formación reactiva de hueso subperióstico con aspecto radiológico en torre ${ }^{1}$.

Debido al escaso número de «turret exostosis» en la literatura y al diagnóstico diferencial con tumores benignos como el osteocondroma o la lesión de Nora, presentamos un nuevo caso.

\section{CASO CLÍNICO}

Mujer de 30 años que consultó por aumento de volumen y dolor al tacto en cara dorsolateral distal del quinto metacarpiano derecho, mano dominante. Un año antes sufrió un golpe directo con un objeto romo en dicha localización, permaneciendo asintomática durante los nueve meses siguientes, notando posteriormente un progresivo aumento de volumen, doloroso al 


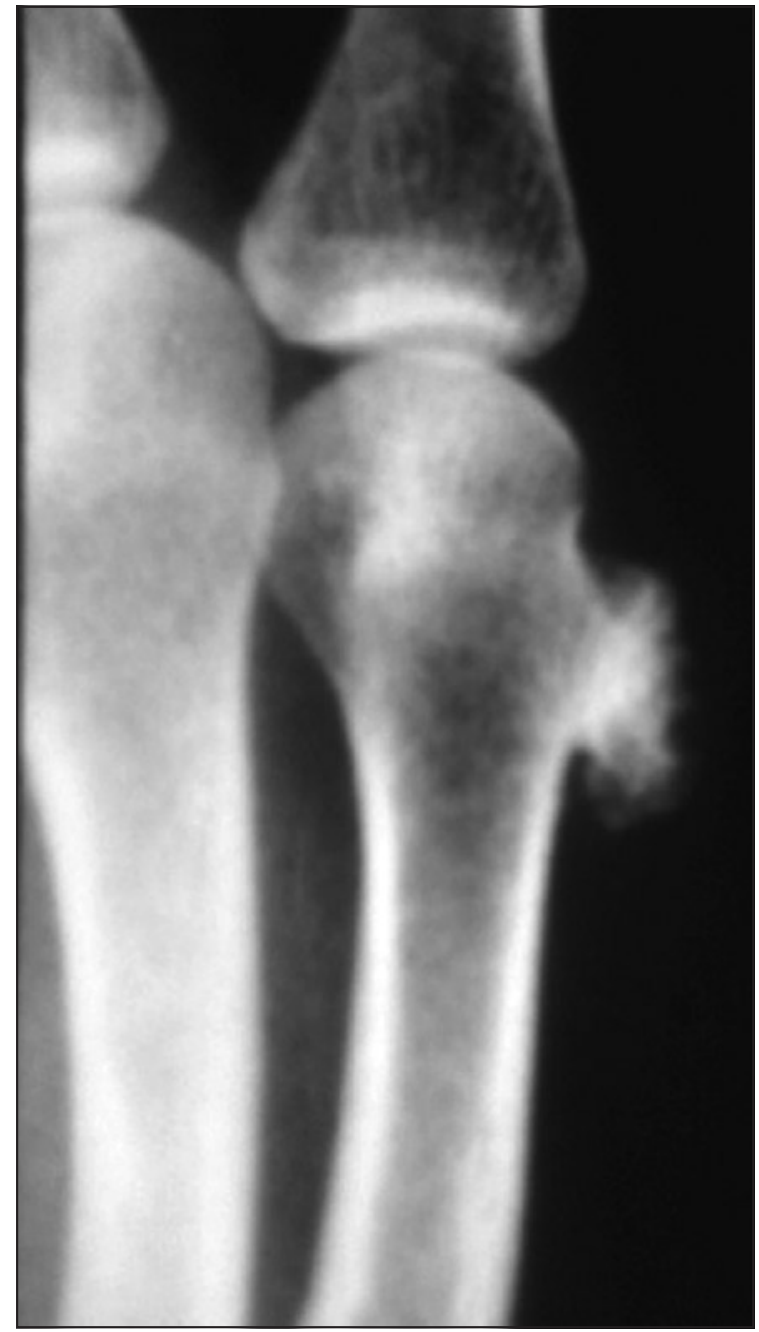

Figura 1: Radiografía anteroposterior de mano en el que se muestra lesión extracortical osificada de cuello de quinto metacarpiano «turret exostosis».

tacto, adherido a planos profundos, sin compromiso cutáneo, de $1 \times 1 \mathrm{~cm}$ de diámetro, que provocaba deformidad del quinto metacarpiano sin repercusión funcional a la flexo-extensión metacarpofalángica, sin pérdida sensitivo-motora en dicha zona. Las radiografías, en proyección anteroposterior y lateral del quinto metacarpiano, revelaron una formación ósea yuxtacortical en el borde cubital distal de la cabeza y cuello del quinto metacarpiano derecho (Figura 1). Se practicó la resección de la exóstosis y los estudios anatomopatológicos informaron sobre la presencia de un tejido óseo reticular con focos de osificación encondral y con trabéculas óseas en aposición directa con el tejido conectivo perióstico. Los controles postoperatorios al año no

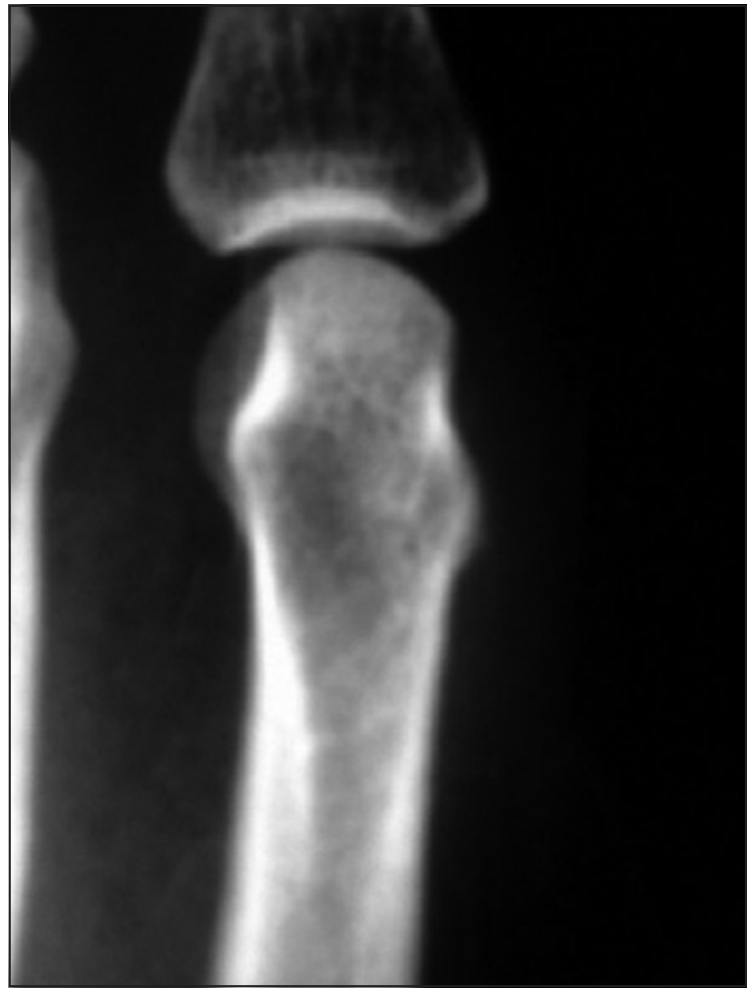

Figura 2: Radiografía anteroposterior de mano al año de la intervención sin recidiva de las exóstosis.

revelaron recidiva y la paciente fue dada de alta sin secuelas (Figura 2).

\section{DISCUSIÓN}

Una «turret exostosis» es una rara complicación tras un traumatismo menor en el dorso de $\operatorname{los} \operatorname{dedos}^{1,2}$. El traumatismo lacera el periostio generando un hematoma subperióstico que posteriormente se osifica. Los dígitos índice y meñique son los más afectados ${ }^{2}$.

Las radiografías realizadas en el momento de la lesión demuestran aumento de partes blandas. La histología revela áreas centrales de hueso trabecular con osificación encondral asociando un aro periférico hipocelular de cartílago. La cortical yuxtalesional siempre está intacta sin identificarse pleomorfismo celular o mitosis atípicas. El diagnóstico diferencial incluye lesiones benignas como el osteocondroma y la lesión de Nora. La primera entidad asienta sobre las metáfisis de los huesos largos siendo la mano una localización infrecuente en cuyo caso asien- 
ta preferentemente en falange proximal, siendo la localización metacarpiana excepcional. La lesión de Nora es una lesión benigna de localización predominante en falange proximal y que en radiografía simple se describe como una masa bien definida de mineralización heterotópica de procedencia perióstica sin presentar cambios en la medular ${ }^{3}$. Las neoplasias malignas a descartar son el osteosarcoma y condrosarcoma. Una historia previa de trauma en la mano y la radiología característica hace más fácil la diferenciación de estas lesiones.

Wissinger publicó 10 casos de hematomas osificados de las falanges ${ }^{1}$. Todas las lesiones eran secundarias a laceración o contusión de las falanges.

El tratamiento de elección de la «turret exostosis» es la resección local ${ }^{4,5}$, debiéndose retrasar la intervención hasta el momento de maduración de la lesión. La mayoría de los autores proponen un periodo de 6 meses desde el trauma inicial hasta la cirugía ${ }^{2,4,5}$. Las radiografías seriadas así como gammagrafías son útiles en determinar la madurez de la lesión². Los márgenes corticales bien definidos en radiografías normales así como el descenso en la captación gammagráfica son indicativos de una lesión madura. Si la resección se practica de forma prematura, existe posibilidad de recidiva. El porcentaje global de recidiva para las exóstosis de los dedos es de un $20 \%$.

En conclusión, una lesión extracortical osificada en región metacarpiana «turret exostosis» tras un traumatismo menor en la mano, debe ser considerada tras los diagnósticos diferenciales de lesiones neoformadoras de hueso.

\section{BIBLIOGRAFÍA}

1. Wissinger HA, Mclain EJ, Boyes JH. Turret exostosis: ossifyng hematoma of the phalanges. J Bone Joint Surg Am, 1966; 48: 105-10.

2. Rubin JA, Steinberg DR. Turret exostosis of the metacarpal: a case report. J Hand Surg Am, 1996; 21: 296-8.

3. Dhondt E, Oudenhoven L, Khan $\mathrm{S}$, et al. Nora's lesion, a distinct radiological entity? Skeletal $\mathrm{Ra}-$ diol, 2006; 35: 497-502.

4. Mohanna M, Moiemen S, Fra- me J. Turret exostosis of the thumb. Br J Plast Surg, 2000; 53: 629-31.

5. Leclere LE, Riccio Al, Helmers SW, et al. Turret exostosis of the talus. Orthopedics, 2010; 13: 33-3. 